\title{
Ventajas y problemas de los métodos automatizados de estudio de susceptibilidad in vitro
}

\author{
PATRICIA GARCÍA C.*
}

\author{
ADVANTAGES AND PROBLEMS OF AUTOMATED \\ ANTIMICROBIAL SUSCEPTIBILITY TESTING
}

\begin{abstract}
Antimicrobial susceptibility studies are an important tool in the successful management of infectious diseases. Automated identification and susceptibility systems have the advantage of earlier results, but they are more expensive than manual conventional ones. Automated systems are less cumbersome and are superior in quality, but there are some technical restrictions with certain microorganisms and antimicrobial agents. In the decision of buying any system, it is necessary to know its advantages in order to justify the high costs of equipment and reagents. An institutional evaluation of its clinical impact is recommended.
\end{abstract}

Key words: Susceptibility test, Automated system, Minimal inhibitory concentration.

En la medicina actual y con el desarrollo importante de resistencia en cocáceas Gram positivas (Staphylococcus, Streptococcus y Enterococcus) y bacilos Gram negativos (enterobacterias y no fermentadores) resulta difícil para el clínico predecir los patrones de resistencia en las infecciones que afectan a los pacientes críticos. ${ }^{1}$ La terapia empírica se inicia generalmente con antimicrobianos de amplio espectro, de alto costo y numerosos efectos adversos. Si el laboratorio de microbiología no entrega resultados oportunos, esta terapia inicial puede prolongarse por más tiempo del necesario.

Es así importante abreviar los tiempos de respuesta por parte del laboratorio, lo que no es posible con los métodos convencionales de estudio de susceptibilidad antimicrobiana. Los sistemas automatizados acortan estos tiempos porque mejoran la sensibilidad analítica de los métodos, es decir, son capaces de detectar el desarrollo bacteriano en una suspensión, con y sin antimicrobianos, antes que el laboratorista pueda detectar turbidez. ${ }^{2}$ Este es el fundamento de los sistemas automatizados de estudio de susceptibilidad, por diferentes métodos (colorimetría, turbidimetría, fluorometría, etc) detectan el desarrollo bacteriano en micropaneles que contienen diluciones seriadas del antimicrobiano, estableciendo la mínima concentración del antimicrobiano que es capaz de inhibir el desarrollo bacteriano

\footnotetext{
* Laboratorio de Microbiología, UDA Laboratorios Clínicos. Facultad de Medicina, Pontificia Universidad Católica de Chile.
} 
Métodos automatizados de estudio de susceptibilidad in vitro - P. García C.

Tabla 1. Sistemas automatizados para identificación y estudio de susceptibilidad bacteriana según el método de detección, marca registrada y fabricante

\begin{tabular}{lll}
\hline Método de detección & Equipo & Fabricante \\
\hline Colorimetría & Vitek ${ }^{\circledR}$ & bioMerieux \\
\hline Turbidimetría & MicroScan ${ }^{\circledR}$ & Dade \\
& Unisept ${ }^{\circledR}$ & API \\
& Pasco ${ }^{\circledR}$ & DIFCO \\
& Sceptor ${ }^{\circledR}$ & Becton Dickinson \\
& Phoenix ${ }^{\circledR}$ & Becton Dickinson \\
\hline Fluorometría & Vitek ${ }^{\circledR}$ & bioMerieux \\
& Sensititre ${ }^{\circledR}$ & Radiometer \\
& MicroScan & Dade \\
& Walk/Away ${ }^{\circledR}$ & \\
\hline
\end{tabular}

(CIM). La Tabla 1 muestra los diferentes métodos de detección que poseen los distintos sistemas automatizados y sus marcas registradas.

\section{Ventajas de los sistemas automatizados de estudio de susceptibilidad in vitro}

La principal ventaja de estos sistemas es la rapidez con que los resultados están disponi- bles. Al menos tres estudios prospectivos, aleatorios y controlados, han comprobado que el abreviar el tiempo de respuesta en los estudios de susceptibilidad in vitro permite un inicio o un cambio más oportuno de la terapia antimicrobiana, lo que conlleva a una reducción en el número de exámenes, reducción en los días de hospitalización, reducción en el uso de antimicrobianos y se traduce además en una importante reducción de los costos totales. ${ }^{3-5}$

Tabla 2. Ventajas de los sistemas automatizados de estudio de susceptibilidad bacteriana

\begin{tabular}{ll}
\hline \multicolumn{1}{c}{ Ventajas } & \multicolumn{1}{c}{ Especificación } \\
\hline Impacto clínico & $\begin{array}{l}\text { Rapidez en el informe de resultado (3-10 hrs vs } \\
16-24 \mathrm{hrs} \text { ). }\end{array}$ \\
& $\begin{array}{l}\text { Inicio o cambio precoz del antimicrobiano. } \\
\text { Reducción de costos: días de hospitalización, exáme- } \\
\text { nes laboratorio. }\end{array}$ \\
\hline Estandarización y control de calidad & $\begin{array}{l}\text { Aumento de la reproducibilidad intra e inter- } \\
\text { laboratorio }\end{array}$ \\
\hline Disminución de la carga de trabajo & Requiere menos personal en métodos que determi- \\
& nan concentración inhibitoria mínima. \\
\hline Disminución de errores post-analíticos & $\begin{array}{l}\text { Evita la transcripción errónea de resultados por dis- } \\
\text { poner programas de comunicación bi-direccional. }\end{array}$ \\
\hline Utilización de sistema de expertos & $\begin{array}{l}\text { Permite actualizar anualmente las guías NCCLS que } \\
\text { permite el informe selectivo de los antimicrobianos }\end{array}$ \\
\hline Patrones fenotípicos de resistencia & $\begin{array}{l}\text { Permite sospechar la presencia de } \beta \text { lactamasas de } \\
\text { espectro extendido y otras } \beta \text { lactamasas. }\end{array}$ \\
\hline Facilita la obtención de estadísticas & $\begin{array}{l}\text { Capacidad de almacenar y procesar la información } \\
\text { para un análisis de tendencias anuales de susceptibi- }\end{array}$ \\
\hline Facilita el control en el uso de antimicrobianos & Informe de los resultados en línea con farmacia. \\
\hline
\end{tabular}


Por otro lado, la implementación de todo sistema automatizado confiere una mejor estandarización y reproducibilidad de los resultados y disminución de la carga de trabajo en los laboratorios ya que los métodos de determinación de CIM (dilución en agar o dilución o microdilución en caldo) son muy laboriosos cuando se realizan manualmente: requieren disponer del antimicrobiano puro con potencia conocida, la preparación de al menos 4 diluciones del fármaco a ensayar y exigen destinar bastante tiempo a la lectura e informe de los resultados, si bien ahora la mayoría de los sistemas manuales de microdilución en caldo traen incorporado un programa computacional que facilita la lectura y la interpretación de los resultados según versiones actualizadas de los criterios NCCLS. ${ }^{6,7}$

Otra importante ventaja es que disminuyen los errores post-analíticos, ya que la conexión en red con el sistema de información de exámenes evita la transcripción de los resultados de un informe que es complejo; además disponen de un sistema de expertos que permite utilizar algoritmos de informes y monitorizar resultados inconsistentes. Estos sistemas de expertos permiten sospechar patrones fenotípicos de resistencia como $\beta$ lactamasas de espectro extendido y cefalosporinasas, ${ }^{8-10}$ facilitan la obtención de estadísticas y permiten el análisis de las tendencias locales de resistencia. Por otra parte, muchos de estos sistemas tienen asociados programas con conexión en red a la farmacia de hospital por lo que puede correlacionarse la susceptibilidad bacteriana con el uso de un determinado antimicrobiano.

Las ventajas de los sistemas automatizados se resumen en la Tabla 2.

\section{Desventajas de los sistemas automatizados de estudio de susceptibilidad in vitro}

El principal inconveniente de estos sistemas es el alto costo en insumos (aproximadamente 3 veces el valor de los métodos manuales), lo que dificulta su implementación en países en vías de desarrollo, si bien algunos países sudamericanos han logrados sustentar los costos en base a tarifas diferenciadas para estudios de susceptibilidad manuales, de los automatizados. Otra buena alternativa para estos sistemas es que cada centro diseñe algoritmos de trabajo donde estos estudios se reserven para situaciones especiales: cuando los métodos manuales

Tabla 3. Inconvenientes de los sistemas automatizados de estudio de susceptibilidad bacteriana

\begin{tabular}{ll}
\hline \multicolumn{1}{c}{ Desventajas } & \multicolumn{1}{c}{ Especificación } \\
\hline Alto costo & $\begin{array}{l}\text { El equipamiento y los insumos (paneles o tarjetas) } \\
\text { son de alto costo }\end{array}$ \\
\hline Requieren método de respaldo & $\begin{array}{l}\text { Frente a falla en el equipo es necesario disponer de un } \\
\text { equipo de respaldo o de una metodología manual de } \\
\text { respaldo. }\end{array}$ \\
\hline No existen normas NCCLS para & $\begin{array}{l}\text { Sin embargo deben considerarse los métodos em- } \\
\text { pleados (puntos de corte o CIM). }\end{array}$ \\
\hline sistemas automatizados & $\begin{array}{l}\text { Paneles o tarjetas estás determinadas por el fabri- } \\
\text { cante. Existen tarjetas especiales pero requieren de } \\
\text { un consumo mínimo. }\end{array}$ \\
\hline ensayados & $\begin{array}{l}\text { Problemas con algunos fármacos y antimicrobianos: } \\
\text { cefepime y Pseudomonas, vancomicina y } \\
\text { Enterococcus, imipenem y bacilos Gram negativos } \\
\text { no fermentadores. }\end{array}$ \\
\hline Discrepancia con métodos de referencia & \\
\hline
\end{tabular}


no entregen resultados fidedignos o se requiera resultados en plazos cortos, como es el caso de pacientes críticos.

Otros inconvenientes son que requieren equipos o métodos de respaldo ante la eventualidad de fallas mecánicas que inhabiliten su uso, que no existen normas para sistemas automatizados (NCCLS), que las tarjetas o paneles tienen un diseño no flexible de los antimicrobianos ensayados, y que no son aplicables a todas las bacterias. Presentan discrepancias con los métodos convencionales o están aún en evaluación, esto es válido para bacterias fastidiosas como Haemophilus influenzae, Streptococcus pneumoniae, ${ }^{11}$ para algunos bacilos Gram negativos no fermentadores, para anaerobios y para algunos antimicrobianos específicos: cefepime en Pseudomonas aeruginosa ${ }^{12,13}$ falsas resistencias o falsas susceptibilidades a imipenem en Pseudomonas aeruginosa y Acinetobacter baumannii ${ }^{14}$ o Enterococcus de susceptibilidad intermedia a vancomicina. ${ }^{15-17}$ Es conocido también que algunas $\beta$ lactamasas inducibles en bacilos Gram negativos requieren una incubación más prolongada para expresar la resistencia. Respecto de estas discrepancias, la FDA ha recomendado que para errores mayores (que el sistema automatizado informe resistencia siendo la cepa susceptible) la tasa no sea mayor que $1,5 \%$, que la concordancia sea a lo menos de $90 \%$ y que las fallas de crecimiento no superen el $10 \% .^{2}$ Las desventajas se resumen en la Tabla 3.

Finalmente, en la decisión de implementar o no estos sistemas automatizados, cada centro debe considerar los factores antes expuestos, donde el ítem costos debe ser siempre considerado en relación al beneficio de disponer los resultados oportunos y de la capacidad de tomar decisiones en el cambio de terapia en el mismo momento que los resultados están disponibles. Del mismo modo la inversión en automatización requiere que el informe de los resultados sea computacional en línea con el médico que requiere un informe oportuno; por lo anterior se recomienda que previo a la implementación de estos sistemas, se realice una evaluación institucional del real impacto clínico y económico.

\section{RESUMEN}

Los estudios de susceptibilidad constituyen una herramienta de gran utilidad en la terapia exitosa de un paciente infectado. Los sistemas automatizados de identificación y estudio de susceptibilidad ofrecen como principal ventaja la disminución del tiempo requerido en la obtención de los resultados; sin embargo, son de mayor costo que los sistemas manuales convencionales. Los sistemas automatizados presentan además otras ventajas que facilitan y mejoran la calidad técnica de los estudios de susceptibilidad; no obstante, se han descrito restricciones técnicas a su uso para determinados microorganismos y antimicrobianos. Es necesario conocer las ventajas y problemas de estos sistemas y considerarlos al momento de decidir la adquisición de alguno de estos sistemas y que podrían justificar el alto costo de equipamiento y en los insumos, aunque también es recomendable una evaluación local del real impacto clínico que estos sistemas pueden presentar.

\section{BIBLIOGRAFÍA}

1.- JORGENSEN J H. Selection criteria for an antimicrobial susceptibility testing system. J Clin Microbiol 1993; 31: 2841-4.

2.- FERRARO M J, JORGENSEN J H. Susceptibility testing instrumentation and computarized expert system for data analysis and interpretation. In Murray PR, Baron E J, Pfaller M A, Tenover F C \& Yolken R H. Manual of Clinical Microbiology, $7^{\text {th }}$ ed 1999 , ASM Press Washington DC, pp. 1593-600.

3.- THENHOLME G M, KAPLAN R L, KARAKUSIS $\mathrm{P} \mathrm{H}$ et al. Clinical impact of rapid identification and susceptibility testing of bacterial blood culture isolates. J Clin Microbiol 1989; 27: 1342-5.

4.- DOERN G V, VAUTOUR R, GAUDET M, LEVY B. Clinical impact of rapid in vitro susceptibility testing and bacterial identification. J Clin Microbiol 1994; 32: 1757-62.

5.- BARENFANGER J, DRAKE C, KACICH G. Clinical and financial benefits of rapid bacterial identification and antimicrobial testing. J Clin Microbiol 1999; 37: 1415-8.

6.- SHETTY N, HILL G, RIDGWAY G L. The Vitek analyser for routine bacterial identification and susceptibility testing: protocols, problems, and pitfalls. J Clin Pathol 1998; 51: 316-23.

7.- McGREGOR A, SCHIO F, BEATON S, BOULTON 
V, PERMAN M, GILBERT G. The Microscan Walkaway diagnostic microbiology system - an evaluation. Pathology 1995; 27: 172-6.

8.- THOMSON K, SANDERS C, SMITH MOLAND E. Use of microdilution panels with and without $\beta$-lactamase inhibitors as a phenotypic test for $\beta$-lactamase production among Escherichia coli, Klebsiella spp., Enterobacter spp., Citrobacter freundii, and Serratia marcescens. Antimicrob Agents Chemother 1999; 43: 1393-400.

9.- SANDERS C, BARRY A, WASHINGTON J et al. Detection of extended-spectrum- $\beta$-lactamaseproducing members of the family Enterobacteriaceae with the Vitek ESBL test. J Clin Microbiol 1996; 34 : 2997-3001.

10.- HADZIYANNIS E, TUOHY M, THOMS L, PROCOP G, WASHINGTON J, HALL G. Screening and confirmatory testing for extended spectrum $\beta$-lactamases (ESBL) in Escherichia coli, Klebsiella pneumoniae, and Klebsiella oxytoca clinical isolates. Diag Microbiol Infect Dis 2000; 36: 113-7.

11.- JORGENSEN J, BARRY A, TRACZEWSKI M, SAHM D, MCELMEEL M, CRAWFORD S. Rapid automated antimicrobial susceptibility testing of Streptococcus pneumoniae by use of the bioMerieux Vitek2. J Clin Microbiol 2000; 38: 2814-8.

12.- JONES R, BIENDENBACH D, MARSHALL S, PFALLER M, DOERN G. Evaluation of the Vitek system to accurately test the susceptibility of Pseudomonas aeruginosa clinical isolates against cefepime. Diag Microbiol Infect Dis 1998; 32: 10710.

13.- BIEDENBACH D J, MARSHALL S A, JONES R N. Accuracy of cefepime antimicrobial susceptibility testing results for Pseudomonas aeruginosa tested on the MicroScan Walk Away system. Diag Microbiol Infect Dis 1999; 33 (4): 305-7.

14.- TSAKRIS A, PANTAZI A, PORNARAS $S$ et al. Pseudo-outbreak of imipenem-resistant Acinetobacter baumannii resulting from false susceptibility testing by a rapid automated system. J Clin Microbiol 2000; 38: 3505-7.

15.- TENOVER F, SWENSON J, O`HARA C, STOCKER S. Ability of commercial and reference antimicrobial susceptibility testing methods to detect vancomycin resistance in Enterococci. J Clin Microbiol 1995; 33: 1524-7.

16.- ENDTZ H, VAN DEN BRAAK N, VAN RFIKHM A et al. Comparison of eight methods to detect vancomycin resistance in Enterococci. J Clin Microbiol 1998; 36: 592-4.

17.- VAN DEN BRAAK N, GOESSENS W, VAN BELKUM A, VERBRUGH H, ENDTZ H. Accuracy of the Vitek 2 system to detect glycopeptide resistance in Enterococci. J Clin Microbiol 2001; 39: 351-3.

Correspondencia a:

Patricia García Cañete

E-mail:pgarcia@med.puc.cl 